\title{
Accretion and ejection in black-hole X-ray transients
}

\author{
N. D. Kylafis ${ }^{1,2}$ and T. M. Belloni ${ }^{3}$ \\ ${ }^{1}$ University of Crete, Physics Department \& Institute of Theoretical \& Computational Physics, 71003 Heraklion, Crete, Greece \\ e-mail: kylafis@physics.uoc.gr \\ 2 Foundation for Research and Technology-Hellas, 71110 Heraklion, Crete, Greece \\ ${ }^{3}$ INAF - Osservatorio Astronomico di Brera, via E. Bianchi 46, 23807 Merate (LC), Italy
}

Received 3 October 2014 / Accepted 20 December 2014

\section{ABSTRACT}

\begin{abstract}
Context. A rich phenomenology has been accumulated over the years regarding accretion and ejection in black-hole X-ray transients (BHTs) and it needs an interpretation.

Aims. Here we summarize the current observational picture of the outbursts of BHTs, based on the evolution traced in a hardnessluminosity diagram (HLD), and we offer a physical interpretation.

Methods. The basic ingredient in our interpretation is the Poynting-Robertson cosmic battery (PRCB), which provides locally the poloidal magnetic field needed for the ejection of the jet. In addition, we make two assumptions, easily justifiable. The first is that the mass-accretion rate to the black hole in a BHT outburst has a generic bell-shaped form, whose characteristic time scale is much longer than the dynamical or the cooling ones. This is guaranteed by the observational fact that all BHTs start their outburst and end it at the quiescent state, i.e., at very low accretion rate, and that state transitions take place over long time scales (hours to days). The second assumption is that at low accretion rates the accretion flow is geometrically thick, ADAF-like, while at high accretion rates it is geometrically thin. Last, but not least, we demonstrate that the previous history of the system is absolutely necessary for the interpretation of the HLD.

Results. Both, at the beginning and the end of an outburst, the PRCB establishes a strong poloidal magnetic field in the ADAF-like part of the accretion flow, and this explains naturally why a jet is always present in the right part of the HLD. In the left part of the HLD, the accretion flow is in the form of a thin disk, and such a disk cannot sustain a strong poloidal magnetic filed. Thus, no jet is expected in this part of the HLD. Finally, the counterclockwise traversal of the HLD is explained as follows: all outbursts start from the quiescent state, in which the inner part of the accretion flow is ADAF-like, threaded by a poloidal magnetic field. As the accretion rate increases and the source moves to the hard state, the poloidal magnetic field in the ADAF forces the flow to remain ADAF and the source to move upwards in the HLD rather than to turn left. Thus, the history of the system determines the counterclockwise traversal of the HLD. As a result, no BHT is expected to ever traverse the entire HLD curve in the clockwise direction.

Conclusions. We offer a physical interpretation of accretion and ejection in BHTs with only one parameter, the mass transfer rate, plus the history of the system.
\end{abstract}

Key words. accretion, accretion disks - black hole physics - magnetic fields - stars: black holes - stars: jets - X-rays: binaries

\section{Introduction}

In the past decade, it has become clear that in order to characterise the spectral evolution of a black-hole X-ray transient (BHT) it helps to produce a hardness-luminosity diagram (HLD). Although separate outbursts even from the same source can look very different, the HLD shows a much clearer phenomenology, with most sources exhibiting a q-shaped curve, traveled counterclockwise, with additional excursions between soft and hard states (see Fig. 1, top panel; Homan et al. 2001; Belloni et al. 2005; Homan \& Belloni 2005; Gierliński \& Newton 2006; Remillard \& McClintock 2006; Fender et al. 2009; Motta et al. 2009; Belloni 2010; Munoz-Darias et al. 2011a; Stiele et al. 2011; Kylafis \& Belloni 2014). The fact that the diagram closes only at its end (lower right) indicates the presence of hysteresis: the source follows a different path in going from $\mathrm{A}$ to $\mathrm{C}$ than it does in going back from $\mathrm{C}$ to $\mathrm{A}$. The presence of hysteresis in the outburst of BHTs was already noticed in the pioneering work by Miyamoto et al. (1995). Adding fast time variability to the picture in the form of root mean square (rms) integrated over a broad range of frequencies, two more useful diagrams have been introduced: the hardness-fractionalrms-variability diagram (HRD; Fig. 1, bottom panel; Belloni et al. 2005) and the absolute-rms-variability-intensity diagram
(RID; Fig. 2; Munoz-Darias et al. 2011a,b). Interestingly, looking at Figs. 1 and 2 one can see that two diagrams show clear hysteresis, but the HRD does not: the hard to soft branch overlaps the soft to hard one. From these diagrams, together with a more detailed view of the fast time variability in terms of power density spectra (PDS) a set of well defined states has been defined (Belloni et al. 2005, 2011; Belloni 2010). These are the quiescent state (QS), the hard state, for historical reasons called low/hard state (LHS), the hard intermediate state (HIMS), the soft intermediate state (SIMS), and the soft state, historically called high/soft state (HSS). The reason for the high/low in the state names is due to the fact that in the early observations, mostly of Cygnus X-1, the HSS was systematically brighter than the LHS at energies below $10 \mathrm{keV}$. However, at higher energies the situation is reversed. As we will show below, the idea that the HSS is observed systematically at higher accretion rate than the LHS persists, despite the fact that the observations of the last decade have shown otherwise. A different classification scheme based on precise measurements of spectral and timing properties was introduced by Remillard \& McClintock (2006) and revised in McClintock et al. (2009). A comparison between the two classification schemes has been provided by Motta et al. (2009). For the purposes of this paper, we will use the first classification. 


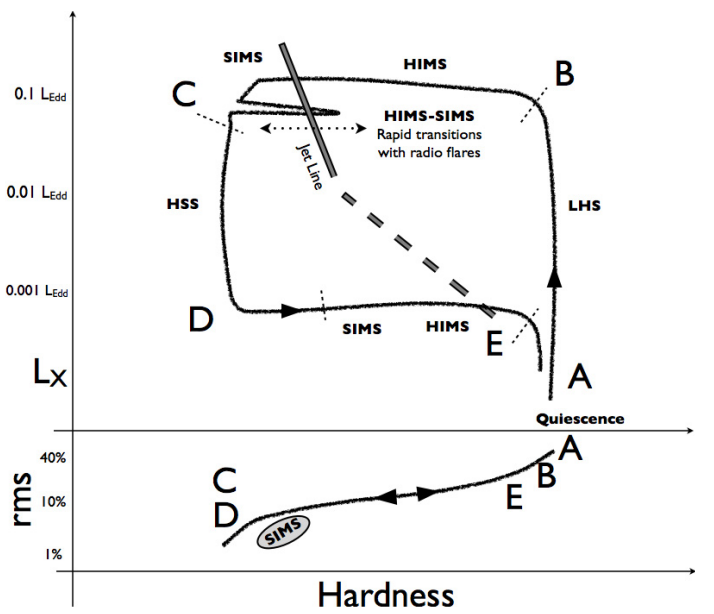

Fig. 1. Schematic representation of the q-shaped curve in a HLD (top panel) and HRD (bottom panel) for black-hole X-ray binaries. The dotted lines in the top panel indicate the transition between states (marked with their acronyms). The arrows give the direction of motion along the line. The jet line is shown as a grey line in the HLD, dashed in the region where it might extend. The letters $\mathrm{A}, \mathrm{B}, \mathrm{C}, \mathrm{D}$, and $\mathrm{E}$ indicate turning points described in the text.

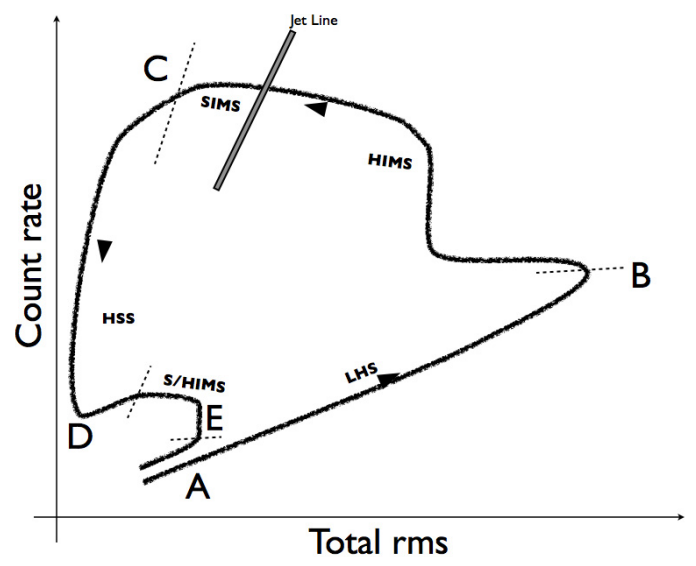

Fig. 2. Schematic representation of the curve traversed by black-hole $\mathrm{X}$-ray binaries in a RID. The dotted lines indicate the transition between states (marked with their acronyms). The arrows give the direction of motion along the line. The jet line is shown as a grey line. The points A, B, C, D, and E mark the same points as in Fig. 1.

In a simplified and generic model, it is assumed that for all states during an outburst the accretion flow is composed of a cool (maximum temperature $\lesssim 1 \mathrm{keV}$ ) geometrically thin and optically thick accretion disk as described by Shakura \& Sunyaev (1973), plus a hot corona near the black hole (the socalled "sombrero" configuration, see Gilfanov 2010). The spectral state is determined by the relative size of the two physical components. In the LHS the corona is large, pushing the accretion disk farther out, in the HSS the disc extends all the way to the innermost stable circular orbit (ISCO) and the corona is correspondingly much smaller in size.

A more realistic model was introduced by Esin et al. (1997, 1998, 2001; see also Narayan et al. 1996, 1997), where the generic corona was replaced by a more physical advection dominated accretion flow (ADAF; Narayan \& Yi 1994, 1995; Abramowicz et al. 1995). In our interpretation of the q-shaped curve, we will start from the Esin et al. (1997) model and will expand it to take into account the wealth of new information that has become available since then.
One important aspect of the q-path in the HLD beyond the presence of hysteresis, which is usually not considered, is its time direction. As we said, the curve is traversed counterclockwise: what prevents the opposite case, a clockwise path? In other words, why is the hard-to-soft transition always at a higher luminosity/accretion rate than the reverse transition (although both can be at different levels during different outbursts)? It is important to understand what breaks the time symmetry. Not all sources exhibit a clean q-curve like the one shown in Fig. 1 (based on the bright transient GX 339-4, see Belloni et al. 2005), but many do and we do not know a single case of a clockwise path. We will use GX 339-4 as the prototype.

For our interpretation, we make two basic assumptions:

1. The accretion rate into the black hole has a generic bellshaped curve as a function of time. By this we mean that the source will start from a very low accretion rate, increase steadily to a sizeable fraction of the Eddington rate, then decrease again down to a very low value. This is clearly justified by the fact that sources start and end their outbursts at very low luminosity (in the QS) and become brighter during the outburst. We do not mean that the accretion rate versus time can always be fitted with a Gaussian function. It can certainly have local maxima and minima. In addition, we assume that the characteristic time scale in this bell-shaped curve is much longer than the dynamical or cooling time scales. This is justified by the fact that state transitions take place over long timescales, typically days.

2. At high accretion rate, the accretion flow is geometrically thin and optically thick as in the solution of Shakura \& Sunyaev (1973), while at low accretion rate it is an ADAF (Narayan \& Yi 1994, 1995; Abramowitz et al. 1995) or ADAF-like.

These two assumptions are commonly accepted and have been verified through numerical simulations (Ohsuga et al. 2009).

Seen with the increased knowledge available now, the Esin et al. (1997) model has some shortcomings. In 1997, our picture was largely based on the results obtained on very few transients by the Ginga satellite (see Tanaka \& Lewin 1995). Their picture featured a very high state (VHS) which appeared at accretion rates higher than those of the HSS, while we know now that the intermediate states (HIMS and SIMS) can appear over the same broad range of luminosities as the HSS. More important, their picture was "one-dimensional", in the sense that there was no hysteresis in the model. The source states were sorted according to accretion rate: from QS to LHS to intermediate to HSS to VHS (see their Fig. 1, where the states are shown next to a vertical arrow indicating accretion rate). What is already clear by looking at the HLD is that dynamically this picture is correct: neglecting the complications introduced by intermediate states, the transition from hard to soft takes place as the accretion rate increases and the reverse transition as the accretion rate decreases. However, statically it is clear that you can have a HSS (point D in Fig. 1) at much lower accretion rate than a LHS (point B in Fig. 1). Finally, the presence of jets, their formation and their destruction were not known at the time and therefore were not part of the model. Here, we extend the model of Esin et al. (1997) and its derivations (see Done et al. 2007, for a review) in order to account for the following:

- The return path (C-D-E in Fig. 1). The outburst decay has been studied in detail through several observational campaigns (see Kalemci et al. 2001, 2003, 2004, 2005, 2006), but to our knowledge it has not been included in theoretical models. 


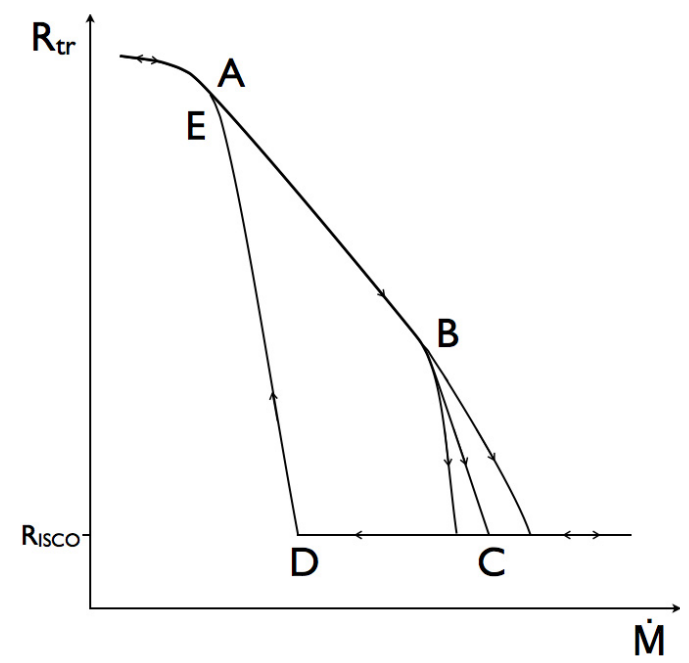

Fig. 3. Schematic representation of the transition radius $R_{\mathrm{tr}}$ between the inner $\mathrm{ADAF}$ and the outer thin disk as a function of mass-accretion rate $\dot{M}$. The points $\mathrm{A}, \mathrm{B}, \mathrm{C}, \mathrm{D}$, and $\mathrm{E}$ mark the same points as in Figs. 1 and 2 . The arrows indicate the direction of motion along the lines.

- Jet formation, destruction, and re-formation. We will describe a novel mechanism for the generation of magnetic field in the ADAF part of the flow, which leads to an explanation for the three main observational facts: i) the formation and evolution of the compact jets observed in quiescence, hard, and hard intermediate states; ii) the eruptive disappearance of the jet once the jet line is crossed (roughly coincident with the HIMS-SIMS transition, see Fender et al. 2009); and iii) the gradual reappearance of the compact jet as the source reaches point $\mathrm{E}$ in Fig. 1.

We will show that only one parameter, the mass accretion rate, plus the history of the system, are sufficient to account for the full evolution of an outburst.

A crucial parameter, elusive to measure, is the transition radius $R_{\text {tr }}$ (Esin et al. 1997) between the outer geometrically thin thermal disk and the inner ADAF portion of the accretion flow. This radius is a function of accretion rate. Theoretical work on $R_{\mathrm{tr}}$, which included evaporation, was presented by Liu et al. (1999; see also Meyer et al. 2000; Meyer-Hofmeister \& Meyer 2001; Qian et al. 2007; Meyer-Hofmeister et al. 2009). Following this model, we show in Fig. 3 a sketch of the dependence of $R_{\text {tr }}$ with mass accretion rate $\dot{M}$. In the QS, $\dot{M}$ is small and $R_{\mathrm{tr}}$ is large. Between points $\mathrm{A}$ and $\mathrm{B}$, in the LHS, $\dot{M}$ increases and $R_{\text {tr }}$ decreases. Between points $\mathrm{B}$ and $\mathrm{C}$, during the intermediate states, $\dot{M}$ continues to increase and $R_{\mathrm{tr}}$ decreases down to $R_{\mathrm{ISCO}}$. Point $\mathrm{C}$ does not have to correspond to the maximum in $\dot{M}$, although it often does, but any further increase in accretion rate is not accompanied by a change in $R_{\mathrm{tr}}$. In Fig. 3 we show three paths corresponding to three different accretion rate values at C. From point $\mathrm{C}$ the source is in the HSS. As the accretion rate starts to decrease, $R_{\mathrm{tr}}$ remains at $R_{\mathrm{ISCO}}$ until point $\mathrm{D}$, when the HSS interval ends. At point $\mathrm{D}$, the inner accretion disk becomes once again radiatively inefficient and increases its thickness (Das \& Sharma 2013). Approaching point E, $R_{\mathrm{tr}}$ starts increasing and the inner ADAF region becomes larger until the loop closes and the source reaches once more the LHS. Observationally, there is no agreement on the details of the variation of the transition radius as a function of accretion rate (see Sect. 2.3), but it is generally accepted that $R_{\text {tr }}$ is large in the QS and in the LHS, when $\dot{M} \lesssim 0.01 \dot{M}_{\mathrm{E}}$, and is at its minimum in the HSS.
The scheme we present in this work is of qualitative nature, but it relies on theoretical ideas and model calculations which are well accepted. Our description will be on the "zeroth order" effects. We will only offer partial speculations for additional details. Our picture is developed for black holes, but many of its aspects can be applied to neutron-star and white-dwarf binaries (Kylafis et al. 2012).

In Sect. 2 we outline the observational appearance of BHTs in the spectral and the timing domain, in Sect. 3 we describe the general picture in order to model the "zeroth order" phenomenology, in Sect. 4 we make some remarks, and in Sect. 5 we present our conclusions.

\section{Spectral and timing properties of BHTs}

Here we present the spectral and timing properties of BHTs, which we will try to interpret below.

\subsection{X-ray spectrum}

The soft X-ray spectra (observed in the HSS) of BHTs are well interpreted with spectra of the form of a multi-temperature blackbody, modified by scattering and relativistic effects, and are generally believed to originate from a geometrically thin and optically thick accretion disk of the type proposed by Shakura \& Sunyaev (1973). For a detailed analysis of the spectra see, e.g., Davis et al. (2005, 2006).

In the LHS, hard spectra are observed and there is much less agreement about their origin, although a general consensus is forming (see below). Models involving a corona (see Gilfanov 2010) posit that the spectra are generated through Comptonization of soft seed (disk) photons by a population of thermal or non-thermal (or hybrid) electrons located in the corona. This class of models as such cannot account for the hysteresis described above.

All BHTs show radio emission that points toward evidence of the presence of a compact radio jet when they are in their QS, LHS and HIMS (see, e.g., Fender \& Belloni 2012). Since in our picture the jet originates from an ADAF, which is associated with the generation of the observed hard spectra, it is natural to speculate that the jet might play a role too. A simple jet model was shown to reproduce the X-ray spectra in the LHS and HIMS (Reig et al. 2003). The same is true for ADAF models (see Done et al. 2007, for a review). Given the presence of a jet and an ADAF, there is no need to introduce a corona, either thermal or non-thermal, to explain the LHS and HIMS hard spectra up to a few hundred keV. In fact, ADAF and jet models can address observational facts that are not included in corona models (Giannios et al. 2004; Giannios 2005; Kylafis et al. 2008; Ingram et al. 2009; Ingram \& Done 2011, 2012). The upscattering of soft photons, which generates the hard spectrum, can take place at the base of the jet (Markoff et al. 2005). However, in this scenario the photons will not be confined to the base of the jet, but will interact with the full compact jet. A model considering the full jet as scattering region fits the broad-band spectra from radio to X-rays (Giannios et al. 2004; Giannios 2005).

In view of what has been outlined above, in this work we will assume that the hard spectrum observed in the LHS and HIMS is produced by Comptonization of soft photons in the ADAF and possibly in the compact jet. The two possibilities are not that different, since the jet originates from the ADAF, which means that at "zeroth order" the mean electron energy in the ADAF and the jet must be comparable.

In the soft states, HSS and SIMS, in addition to the thermal disk, a steep power-law component is observed at high energies, 
whose luminosity is only a small fraction of the total luminosity. This component has not received as much attention. No jet emission from the core of the source has been observed in soft states, which points towards an alternative nature of this emission. A possible origin is through Comptonization in nonthermal flares above and below the thermal disk (Poutanen et al. 1997; Gierliński et al. 1999).

\subsection{Time variability}

Fast time variability is very state-dependent during outbursts of BHTs and it follows a very clear pattern (see Belloni 2010; Belloni et al. 2011). Variations in time-variability properties can be followed in the diagrams described above (Figs. 1 and 2). The total fractional rms can be followed in the HRD (bottom panel of Fig. 1). Here, as already remarked, no hysteresis is visible: the source moves from a high amount of variability (30-40\%) in the LHS down to $\sim 1 \%$ in the HSS following a well defined path passing through the HIMS, and follows the same path on the return to quiescence. The only outliers correspond to the SIMS, which forms a separate "cloud" below the correlation.

The same evolution can be seen in the RLD (Fig. 2), for which no spectral information is needed. Hysteresis is observed in this diagram also. The SIMS is confined in a very small region of the RLD (see Muñoz-Darias et al. 2011a).

\section{Interpretation of the outbursts of BHTs}

Most of the BHT time is spent in the QS (see e.g. Levine et al. 2006), at a low accretion rate and luminosity. Only sensitive instruments like those on board Chandra and XMM-Newton can detect them and for some of them long exposure times are needed (see, e.g., Gallo et al. 2006, and references therein). For reasons which are thought to be connected to ionisation instabilities in the outer regions of the accretion disk (Meyer \& MeyerHofmeister 1981; Smak 1984; for a review see Lasota 2001), at some point a significant amount of matter starts accreting into the black hole and an outburst starts. Since all outbursts (with the exception of the peculiar GRS 1915+105, which started an outburst in 1992 and at the time or writing it is still active) terminate after weeks to months again to the QS, our "zeroth order" assumption of a bell-shaped time evolution of accretion rate is justified. Of course by "bell-shaped" we do not in any way mean that it has a Gaussian shape, but merely that it starts low, increases to a maximum or multiple local maxima, and then decreases again to the same value at which it started. Our additional assumption, that the characteristic time scale for changes in the accretion rate is much larger than the dynamical and cooling time scales of the flow, guarantees that the flow finds quickly its equlibrium.

Through multi wavelength observations, we know that at large distances from the black hole $\left(r \gtrsim 2 \times 10^{4} R_{\mathrm{g}}\right.$, where $R_{\mathrm{g}}=G M / c^{2}$ is the gravitational radius and $M$ is the mass of the black hole) the accreting matter forms a thin disk (McClintock et al. 2003; see also Marsh et al. 1994; Orosz et al. 1994). As per our assumption, the inner region is in an ADAF state and accretion is at a very low level at the beginning of an outburst.

\subsection{Quiescent state}

We start in quiescence, well below point A in Fig. 1, top panel. The accretion rate is very low and the accretion flow inside the transition radius $R_{\text {tr }}$ is well described by an ADAF. Radio emission associated to a compact jet has been observed from BHTs in quiescence (see Gallo et al. 2006). The hard power-law energy spectrum is produced by soft cyclotron photons from the jet upscattered by electrons either in the jet (Giannios 2005) or in the ADAF (Done et al. 2007). A fraction of disk photons, coming from farther out in the accretion flow, are also scattered, but they contribute a small fraction of the flux.

Three-dimensional magnetohydrodynamic simulations (Machida et al. 2006; Hawley 2009; Romanova et al. 2009; Mignone et al. 2010) have shown that there are two mechanisms for jet formation, both requiring a strong large-scale magnetic field: plasma gun/magnetic tower (Contopoulos 1995; LyndenBell 1996), and centrifugal driving (Blandford \& Payne 1982). Such a magnetic field can originate from a large distance and the advecting flow carries it to the inner region and amplifies it (Igumenshchev 2008; Lovelace et al. 2009; Tchekhovskoy et al. 2011). Alternatively, the field can be produced locally in the inner region, a possibility which we favor due to its simplicity and to the fact that no random process is involved. A natural mechanism for the formation of a strong, poloidal magnetic field is the Poynting-Robertson cosmic battery (PRCB, Contopoulos \& Kazanas 1998; see also Contopoulos et al. 2006; Christodoulou et al. 2008). This mechanism is very efficient, because the inner region of the accretion flow is geometrically thick. This means that most of the radiation emitted near the ISCO will contribute to the battery mechanism. As for time scales (see Kylafis et al. 2012), since most of the life of BHTs is in the QS, there is more than sufficient time for the formation of the poloidal field needed for this mechanism to operate.

It is important to stress here the following: the dynamical time scale of the flow is not the fastest one in the problem. The fastest time scale is the one for the diffusion of the magnetic field in the flow. Thus, the magnetic field that is created by the Cosmic Battery diffuses outward in the flow faster than it is advected inwards (Contopoulos et al. 2006). This allows its nonlinear growth.

\subsection{Hard state}

The efficiency of the Cosmic Battery is directly proportional to the luminosity. Therefore, as the source leaves the QS and enters the LHS, increasing its luminosity, the PRCB will produce a stronger magnetic field. The field will be able to support a stronger jet, brighter in the radio (e.g., Giannios 2005). This leads to a positive correlation between radio and X-ray luminosity (see Gallo 2010; Corbel et al. 2013a). We are now at point A in the HLD (Fig. 1). As our source brightens, the inner accretion flow remains ADAF, the PRCB works more efficiently and the $\mathrm{X}$-ray spectrum does not change dramatically in the $2-20 \mathrm{keV}$ band, leading to a nearly vertical line in the HLD, with limited softening (see Motta et al. 2009). However, the upscattering of more and more soft photons cools the electron population (Compton cooling; Done et al. 2007), leading to a decrease in the high-energy cutoff $E_{\mathrm{c}}$ in the spectrum (Motta et al. 2009). The soft photon input increases because the transition radius moves inwards (Esin et al. 1997; Liu et al. 1999; Das \& Sharma 2013) and therefore the thermal component becomes hotter and more luminous. Above $1 \mathrm{keV}$, no direct emission is yet observable, so the only effect of the soft photons is to change the properties of the input photons for Comptonization (Sobolewska et al. 2011).

The ADAF inner flow within the transition radius is permeated by a strong poloidal magnetic filed, near equipartition, at the ISCO (Kylafis et al. 2012), which drops off with radius. This field supports the jet and increases the strength of 
the magneto-rotational instability (Bai \& Stone 2013). Because of this, there is efficient transport of matter inwards and the density remains low. Thus, the source moves upwards in the q curve (towards point B, Fig. 1, top panel) and does not turn left (towards point E). This is where the history of the system plays a crucial role. From quiescence to this time, the inner part of the flow is ADAF-like. This ADAF is permiated by a strong poloidal magnetic field produced locally by the Cosmic Battery. According to the work of Bai \& Stone (2013), this magnetic field forces the source to move upwards in the q-curve, i.e., to traverse it in the counterclockwise direction. Another consequence is that the upcoming state transitions LHS-HIMS-SIMSHSS (from point $B$ to point $C$ in Fig. 1) take place at higher accretion rate than in the return path (from point $\mathrm{D}$ to point $\mathrm{E}$ in Fig. 1). In the return path, the history is completely different (see Sects. 3.6-3.10 below). From point $\mathrm{C}$ to point $\mathrm{D}$ in Fig. 1 the flow is in the form of a Shakura-Sunyaev thin disk and the Cosmic Battery is very inefficient in producing a poloidal magnetic field.

Some authors suggested that in the LHS the transition radius is not located at several tens of gravitational radii, but it is close to the ISCO (Miller et al. 2006a,b). The small-radius scenario for the LHS has been challenged by Done et al. (2007). Recent works also appear to be inconclusive, with support for the smallradius picture (Reis et al. 2010; Reynolds \& Miller 2013) and for the opposite (Plant et al. 2013). We think that the truncated disk is to be preferred, as it explains physically the X-ray spectra and variability (Done et al. 2007; Ingram et al. 2009; Ingram $\&$ Done 2011, 2012) and the jet formation/destruction (Kylafis et al. 2012).

In the LHS, the total variability is large, up to $\sim 40 \%$ fractional rms. As the thin disk can vary only on a longer time scale, corresponding to the viscous time scale (Uttley et al. 2011; Wilkinson \& Uttley 2009), the observed variability must be generated in the hard component which dominates the flux, whether the ADAF or the jet. This was confirmed by Axelsson et al. (2013). As the source brightens and softens, the variability decreases and all characteristic frequencies increase, consistent with them being connected to decreasing radii.

In the HLD, moving from point $\mathrm{A}$ to point $\mathrm{B}$ along the LHS branch, the luminosity increases, the spectral photon index increases from $\sim 1.6$ to $\sim 2.1$, the high-energy cutoff decreases significantly (Motta et al. 2009) and, depending on accretion rate, interstellar absorption and observing X-ray band, the thin disk begins to contribute to the observed photons. At the top, the source turns left and enters the HIMS.

\subsection{Hard intermediate state}

At point B in the HLD (Fig. 1, top panel) the HIMS starts and the source moves left in the diagram, with a softening spectrum. As a function of time, the accretion rate increases and $R_{\mathrm{tr}}$ decreases. The ADAF section of the flow becomes smaller and the thin disk extends closer to the black hole. Therefore, the relative contribution of the two parts of the accretion flow changes and the thin disk contributes more and more. In the energy band above $\sim 1 \mathrm{keV}$, this is enhanced by the fact that the disk becomes hotter and contributes more photons to the detector. As an example, Muñoz-Darias et al. (2011b) observe precisely this effect in MAXI J1659-152.

As the inner ADAF portion of the accretion flow becomes smaller, another effect causes the decrease in power-law luminosity. Since the transition radius $R_{\mathrm{tr}}$ becomes smaller, the jet becomes narrower, its optical depth lowers, Comptonization becomes less efficient and the power law in the energy spectrum steepens (Kylafis et al. 2008). If the Comptonization takes place in the ADAF rather than in the jet, a similar argument can be made. Moreover, as the geometrically thick ADAF shrinks to smaller radii, it will reach a point where the disk will not be able to sustain the magnetic field produced by the PRCB (Kylafis et al. 2012). The jet becomes eruptive and is made of discrete blobs with $\Gamma>2$ (Fender et al. 2004). When the so-called "jet line" is reached (Fender et al. 2004; Fig. 1), the thin disk extends to the ISCO.

As the disk may support energetic flares (Poutanen et al. 1997; Gierliński et al. 1999), the high-energy cutoff $E_{\mathrm{c}}$ is now determined by the non-thermal electrons in the flares. This explains the increase in $E_{\mathrm{c}}$ observed in the HIMS as a function of time (see e.g. Motta et al. 2009). The high-energy spectrum is now of non-thermal nature (see also Grove et al. 1998).

In the timing domain, the PDS is the extension to higher frequencies of that in the LHS. The observed characteristic peaks correspond to those in the LHS, but with higher characteristic frequencies (e.g. Belloni et al. 2011). The characteristic frequencies move in unison (Belloni et al. 2005; notice that in Cyg X-1, a persistent system, one additional component was seen to remain at the same frequency across LHS-HIMS transitions, Pottschmidt et al. 2003). Therefore the system displays effectively one frequency. The amount of variability, measured as integrated fractional variability, is lower than in the LHS $(10-20 \%)$. This can be interpreted within our picture. The thin disk, which shows little variability in this state (Gilfanov 2010), increases its contribution, lowering the total rms (Gierliński $\&$ Zdziarski 2005; Belloni et al., in prep.). Since the variability originates from the ADAF/jet, higher variability is observed at higher energies (see, e.g., Gilfanov 2010; Belloni et al. 2011). Notice that at high energies the spectrum is composed of two contributions: the ADAF and the energetic flares discussed above.

The truncated-disk model, with an inner ADAF and an outer Shakura-Sunyaev disk, explains the variability properties. A prediction of our picture is that in the upper branch of the q-diagram in the HLD (between points B and C in Fig. 1), the average hard time lag should decrease as the source softens, because as the ADAF region becomes smaller the travel time of the upscattered photons decreases.

\subsection{First jet-line crossing}

The jet line (Fig. 1, top panel) is defined as the position in the HLD corresponding to the ejection of a major transient relativistic jet as observed in the radio band (Fender et al. 2004). Its location is close to that of the HIMS-SIMS transition (which is defined by marked and abrupt changes in the timing properties, see Belloni 2010), although an exact correspondence could not be made with the current data, rather sparse in radio (Fender et al. 2009). It also marks the disappearance of the compact jet (see Fender et al. 2004; Miller-Jones et al. 2012). As discussed in Kylafis et al. (2012), at this stage the geometrically thin disk is not able to sustain the magnetic field produced by the PRCB and becomes unstable to non-axisymmetric "Rayleigh-Taylor-type" instability modes. The accumulated magnetic field escapes to the outer disk in the form of magnetic "strands" and this may explain the flaring activity. While the timing properties change radically across the HIMS-SIMS transition, the energy spectrum below 10-20 keV changes only minimally, as recorded by the small change in hardness. A change in the evolution of the high-energy cutoff has however been observed (Motta et al. 2009). The fast 
variability is stronger at high energies, which makes a disk origin unlikely. In our picture, the variability is associated to the invoked magnetic flaring activity above and below the disk.

\subsection{Soft intermediate state}

The HIMS and the SIMS correspond to what in the pre-RXTE era was called Very High State (VHS), and Intermediate State (see Miyamoto et al. 1993; Belloni et al. 1997). Now we know that as the accretion rate increases the sequence of states is LHSHIMS-SIMS-HSS, but the HLD in Fig. 1, where the $y$ axis can be taken as a proxy for accretion rate, shows that the HSS does not correspond necessarily to a higher accretion rate than the LHS. The coverage with Ginga was not sufficient to show this sequence and affected the model of Esin et al. (1997).

In the SIMS we are to the left of the jet line. The state is defined by changes in the PDS. The identifying feature is the presence of the so-called type-B QPO, which has been shown not to be an evolution of the one observed in the HIMS (type-C QPO; see Belloni et al. 2005; Casella et al. 2004, 2005; Motta et al. 2011, 2012). Notice that the SIMS in the HRD is characterised by a drop in total fractional rms (the ellipse in Fig. 1, bottom panel). This is mainly due to the disappearing of the HIMS broad-band components in the PDS, which are replaced by a weaker power-law noise.

\subsection{Soft state}

After the SIMS is crossed, the source enters the HSS (point C in the HLD, Fig. 1). In outbursts like those of the prototypical source GX 339-4, where the HLD is similar to that in Fig. 1, the HSS after the SIMS marks the maximum accretion rate. From point $\mathrm{C}$ on, the accretion rate starts decreasing. However, there are sources where the accretion rate continues to rise even after the HSS is reached. They enter the "anomalous state" (Belloni 2010). We do not discuss this state because we consider it a "first-order effect". It is important to notice however that the anomalous state does not correspond to the VHS of Esin et al. (1997).

In the HSS, the energy spectrum is dominated by a soft thermal component, with the addition of an energetically negligible steep power-law component with $\Gamma>2.4$, which has been observed to extend up to $1 \mathrm{MeV}$ (Grove et al. 1998). In all models, the thermal component is associated to a geometrically thin accretion disk extending all the way to the ISCO, while the hard power law could originate from Comptonization of soft photons by a non-thermal population of electrons in flares above and below the disk. As the mass accretion rate decreases, the accretion disk remains geometrically thin and extends to the ISCO. Since the luminosity decreases, the inner disk temperature also decreases and the hardness decreases (see Motta et al. 2010; Stiele et al. 2012). The source reaches point D.

The low level (a few \%) of fast variability observed in the PDS of the HSS comes from the power-law component and not from the thermal disk, as shown by Churazov et al. (2001), through the analysis of Fourier-resolved energy spectra.

\subsection{Soft intermediate state at low luminosity}

At point $\mathrm{D}$, as the mass accretion rate continues to decrease, the inner portion of the disk becomes again radiatively inefficient and thus geometrically thick (Das \& Sharma 2013), with evaporation playing a significant role (Liu et al. 1999). Simulations have shown that the transition radius between the two parts of the flow (the inner ADAF and the outer thin disk) moves outward as the mass accretion rate decreases further (Das \& Sharma 2013). Therefore, for the lower branch of the q-diagram (point $D$ to point E), when the source hardens again showing limited flux decrease, we have the reverse of what happened for the upper branch (point B to point $\mathrm{C}$ ), with the transition radius $R_{\mathrm{tr}}$ moving outwards with time (see Fig. 3).

We remark here that, as the accretion rate decreases, evaporation of the inner part of the accretion disk and the initiation of an inner ADAF will take place at a more or less specific accretion rate. This implies that the source will always turn right at about point $\mathrm{D}$ (i.e., at a specific accretion rate), independent of how high the luminosity was in the upper branch of the q-shaped curve (points B and C in Fig. 1, top panel). This is exactly what has been seen in the multiple outbursts of GX 339-4 (Motta et al. 2011). With the presence of a geometrically thick ADAF in the inner part of the flow, the PRCB can again operate efficiently in creating the magnetic field necessary for the production of a jet. The SIMS is entered again, as indicated by the appearance of type-B QPOs (Stiele et al. 2011; Motta et al. 2011).

A radio compact jet is observed only later, when the source has reached the hard state, but its formation must have started earlier. A gradual development of the jet has been observed in detail (Corbel et al. 2013b and references therein). Notice that the photon index of the hard component in the SIMS in the lower branch ( 2.1, see Stiele et al. 2011) is lower than that of the upper branch $(\sim 2.4)$.

\subsection{Hard intermediate state at low luminosity}

The SIMS-HIMS transition takes place on the lower branch as the accretion rate decreases and the source hardens. Corresponding to this transition, no eruptive jet similar to those in the upper branch has been observed. We predict that none will be observed, because as the ADAF inner portion of the flow continues to increase, the PRCB will continue working and the jet will build up smoothly. In the lower branch, no instability occurs, although as the jet is formed variability is naturally expected.

The spectral evolution is symmetric to what took place in the upper branch. The ADAF flow inside $R_{\mathrm{tr}}$ adds to the energy spectrum a hard component, deriving from upscattering of softer disk photons in the inner flow or in the forming jet. The optically thick disk part recedes and its temperature decreases, also leading to a hardening of the energy spectrum. This is what was observed by Munoz-Darias et al. (2011b) for MAXI J1659-152.

In our picture, we make the prediction that in the lower branch of the q-shaped curve in the HLD the average hard time lag should increase as the source hardens. This is because the ADAF region, where the Comptonization takes place, increases its radius (Das \& Sharma 2013) and therefore the light travel time for Comptonized photons increases.

\subsection{Second jet-line crossing}

The source is now around point E in the HLD (Fig. 1). As we discussed above, the jet line corresponding to the HIMS-SIMS transition does not have a counterpart at the reverse SIMS-HIMS transition. We define the crossing of the jet line, or its extension at low fluxes, as the time at which the compact radio jet is observed again. This occurs shortly before the full hard state is reached (Miller-Jones et al. 2012). At the time of this reverse crossing, a large part of the inner accretion flow is geometrically 
thick and $R_{\text {tr }}$ is now at tens to hundreds gravitational radii and the energy spectrum is harder than at the first jet line crossing. This leads to a diagonal jet line in the HLD in Fig. 1.

\subsection{Back to the hard state}

The LHS is reached again when the ADAF flow takes most of the X-ray emitting region of the disk. As in the case of the start of the outburst, the seed photons for Comptonization are mainly cyclotron photons from the jet and the Comptonization itself takes place either in the jet itself or in the ADAF, with a small contribution from the disk.

\subsection{Return to the quiescent state}

The accretion rate continues to decrease and the reverse of the initial LHS takes place, with the magnetic field produced by the PRCB becoming weaker, making the jet fainter, with the energy spectrum hardening only marginally. The source goes to quiescence remaining in the LHS and leaving the HLD in Fig. 1 from the bottom side along the same path as at the start.

\section{Remarks}

The curve shown schematically in the HLD (Fig. 1. top panel) is a hysteresis curve, as it is observed in many branches of science. All such curves represent cases when the future development of a system depends on its past history in addition to its present. In the case of black hole binaries, the poloidal magnetic field created by the PRCB made possible by the ADAF in the quiescent and hard states "reminds" the flow that it must remain in an ADAF state, although the accretion rate is increasing. Only at high accretion rate the thermal disk prevails and there is a state transition.

Therefore, the stability of the soft and hard states at the same luminosity level can be described as follows. Let us take an accretion rate such that the observed luminosity puts the source between the upper and lower branches in the HLD. For this accretion rate, two states are possible: LHS on the right branch (ADAF-like) or HSS (Shakura-Sunyaev-like) on the left branch. This is a static view: given only the accretion rate, we cannot state where the source could be found. However, when moving from A to B in Fig. 1, the poloidal magnetic field that necessarily exists at $R_{\mathrm{tr}}$ "reminds" the accretion flow that it should retain its flow efficiency (Bai \& Stone 2013), and therefore remain ADAF, despite the increase of the accretion rate. Thus, the source moves vertically from point A to point B and does not turn left. Similarly, when the source moves from C to D in Fig. 1, radiative efficiency in the disk forces the disk to remain thin.

In summary, the history of the system, in addition to a single parameter (accretion rate) are sufficient to explain qualitatively the hysteresis curve. We remark that no astrophysical models can explain all observational details and our proposed zeroth-order picture is no exception. Future work will address "first-order" effects, such as the presence and nature of the anomalous state (Belloni 2010), the different levels of transition luminosity for multiple outbursts of GX 339-4 (where the q shapes are "nested" within each other, see e.g. Belloni 2010), the repeated HIMSSIMS-HIMS transitions with multiple crossings of the jet line, the sources which do not display such a well behaved q diagram, and so on.

\section{Conclusions}

Making use of a) the novel idea of the Poynting-Robertson Cosmic Battery (Contopoulos \& Kazanas 1998); b) two assumptions easily justifiable; and c) the history of BHTs, we have been able to interpret the major observational properties of the evolution of outbursts of BHTs. The assumptions are:

1. The accretion rate onto the central object has a generic bellshaped curve, starting from a very low level, increasing steadily up to a level that can be a sizeable fraction of the Eddington value, and decreasing again to a very low level. This is justified by the fact that BHTs start and end their outbursts in quiescence, at very low luminosity and that during their outburst they reach a high luminosity, often close to Eddington. In addition, we assume that the time scale for accretion-rate changes is much longer than the dynamical and cooling time scales.

2. At high accretion rates, the accretion flow solution is that of an optically thick and geometrically thin disk (Shakura \& Sunyaev 1973). At low accretion rates, it is radiatively inefficient, geometrically thick, optically thin and advection dominated (ADAF; Narayan \& Yi 1994, 1995; Abramowitz et al. 1995). Both these pictures are widely accepted and have been tested with numerical simulations (Ohsuga et al. 2009).

When the accretion flow possesses an inner ADAF portion, the PRCB works efficiently (Kylafis et al. 2012). Therefore, a strong poloidal magnetic field is formed and a jet can be built on time scales of hours to days, depending on the luminosity. This is what is observed in the HLD: on the right track, the accretion rate varies considerably, a large part of the flow is ADAF-like and a compact jet is present.

The sourses traverse the HLD in the counterclockwise direction, because their history plays a significant role. All outbursts start at low luminosity, when the sources are in the quiescent state. In this state, due to the low accretion rate, the major part of the flow, its inner part, is ADAF-like and the PRCB works efficiently. The higher the accretion rate, the higher the luminosity, the larger the efficiency of the PRCB, and the stronger the poloidal magnetic field that is produced there. Thus, the sources are forced (Bai \& Stone 2013) to move upwards in the HLD (counterclockwise direction) rather than move to the left (clockwise direction).

At high accretion rates, the flow switches to a full ShakuraSunyaev solution. In this case, the PRCB is no longer efficient and is not able to form a strong poloidal magnetic field to keep producing a jet. No jet has been observed from a source in the soft state.

In this picture, it is natural to expect that a transient jet is produced in the first jet line crossing (hard to soft) but not in the second (soft to hard). As the first jet line is approached, the ADAF part of the flow, the one which feeds the jet, shrinks. The geometrically thin disk cannot sustain the existing field and becomes unstable to instability modes of Rayleigh-Taylor type. The accumulated magnetic field then escapes and a flare is produced by magnetic reconnection. During the second jet line crossing, the low luminosity implies that the poloidal magnetic field is built slowly in the ADAF and grows steadily. No transient ejection can be produced in this case.

In summary, we have shown that the main observed features along the q-shaped curve in the HLD can be reproduced with only one physical parameter, the mass accretion rate. Additional ingredients in our picture are the Poynting-Robertson cosmic 
battery (Contopoulos \& Kazanas 1998) and the history of the system. The q-shaped curve will always be traversed in the counterclockwise direction and our prediction is that no clockwise evolution will be seen in the future. In addition, we predict that the average hard time lags decrease with time along the upper branch and increase with time along the lower branch. For the formation and the destruction of the jet, our picture can be applied also to neutron-star and white-dwarf binaries (Kylafis et al. 2012).

Acknowledgements. We thank an anonymous referee for helpful suggestions and comments, which have improved our paper in both content and readability. We have also profited from discussions with Iossif Papadakis. One of us (N.D.K.) acknowledges useful discussions with P. Casella, I. Contopoulos, B. F. Liu, S. Motta, R. Narayan, and A. Zdziarski. This research has been supported in part by the "RoboPol" project, which is implemented under the "ARISTEIA" Action of the "Operational Program Education and Lifelong Learning" and is co-funded by the European Social Fund (ESF) and National Resources. Also, by EU Marie Curie projects No. 39965 and ITN 215212 ("Black Hole Universe"), EU REGPOT project number 206469, a Small Research Grant from the University of Crete, a COST-STSM-MP0905 grant and a grant from the European Astronomical Society in 2012. T.M.B. acknowledges support from INAF-PRIN 2012-6.

\section{References}

Abramowicz, M. A., Chen, X., Kato, S., \& Regev, O. 1995, ApJ, 438, L37 Axelsson, M., Hjalmarsdotter, L., \& Done, C. 2013, MNRAS, 431, 1987 Bai, X.-N., \& Stone, J. M. 2013, ApJ, 767, 30

Belloni, T. 2010, Lect. Notes Phys., 794, 53

Belloni, T., van der Klis, M., Lewin, W. H. G. et al. 1997, A\&A, 322, 857

Belloni, T., Homan, J., Casella, P., et al. 2005, A\&A, 440, 207

Belloni, T., Motta, S., \& Muñoz-Darias, T. 2011, Bull. Astron. Soc. India, 39, 409

Blandford, R. D., \& Payne D. G. 1982, MNRAS, 199, 883

Casella, P., Belloni, T., Homan, J., \& Stella, L. 2004, A\&A, 426, 587

Casella, P., Belloni, T., \& Stella, L. 2005, ApJ, 629, 403

Christodoulou, D. M., Contopoulos, I., \& Kazanas, D. 2008, ApJ, 674, 388

Churazov, E., Gilfanov, M., \& Revnivtsev, M. 2001, MNRAS, 321, 759

Contopoulos, I. 1995, ApJ, 450, 616

Contopoulos, I., \& Kazanas, D. 1998, ApJ, 508, 859

Contopoulos, I., Kazanas, D., \& Christodoulou, D. M. 2006, ApJ, 652, 1451

Corbel, S. Coriat, M., Brocksopp. C., et al. 2013a, MNRAS, 428, 2500

Corbel, S. Aussel, H., Broderick, J. W., et al. 2013b, MNRAS, 431, L107

Das, U., \& Sharma, P. 2013, MNRAS, 435, 2431

Davis, S. W., Blaes, O. M., Hubeny, I., \& Turner, N. J. 2005, ApJ, 621, 372

Davis, S. W., Done, C., \& Blaes, O. M. 2006, ApJ, 647, 525

Done, C., Gierliński, M., \& Kubota, A. 2007, A\&ARv, 15, 1

Esin, A. A., McClintock, J. E., \& Narayan, R. 1997, ApJ, 489, 865

Esin, A. A., Narayan, R., Cui, W., Grove, J. E., \& Zhang, S.-N. 1998, ApJ, 505,854

Esin, A. A., McClintock J. E., Drake, J. J., et al. 2001, ApJ, 555, 483

Fender, R. P., \& Belloni, T. M. 2012, Science, 212, 337

Fender, R. P., Belloni, T. M., \& Gallo, E. 2004, MNRAS, 355, 1105

Fender, R. P., Homan, J., \& Belloni, T. M. 2009, MNRAS, 396, 1370

Gallo, E. 2010, Lect. Notes Phys., 794, 85

Gallo, E., Fender, R. P., Miller-Jones, J. C. A., et al. 2006, MNRAS, 370, 1351

Gilfanov, M. 2010, Lect. Notes Phys., 794, 17

Giannios, D. 2005, A\&A, 437, 1007

Giannios, D., Kylafis, N. D., \& Psaltis, D. 2004, A\&A, 425, 163

Gierliński, M., \& Newton, J. 2006, MNRAS, 370, 837

Gierliński, M., \& Zdziarski, A. A. 2005, MNRAS, 363, 1349

Gierliński, M., Zdziarski, A. A., Poutanen, J., et al. 1999, MNRAS, 309, 496

Grove, J. E., Johnson, W. N., Kroeger, R. A., et al. 1998, ApJ, 500, 899

Hawley, J. F. 2009, Ap\& SS, 320, 107

Homan, J., \& Belloni, T. 2005, Ap\&SS, 300, 107

Homan, J., Wijnands, R., van der Klis, M., et al. 2001, ApJS, 132, 377

Igumenshev, I. V. 2008, ApJ, 677, 317

Ingram, A., \& Done, C. 2011, MNRAS, 415, 2323

Ingram, A., \& Done, C. 2012, MNRAS, 419, 2363

Ingram, A., Done, C., \& Fragile, P. C. 2009, MNRAS, 397, L101

Kalemci, E., Tomsick, J. A., Rothschild, R. E., Pottschmidt, K., \& Kaaret, P. 2001, ApJ, 563, 239
Kalemci, E., Tomsick, J. A., Rothschild, R. E., et al. 2003, ApJ, 586, 419

Kalemci, E., Tomsick, J. A., Rothschild, R. E., Pottschmidt, K., \& Kaaret, P. 2004, ApJ, 603, 231

Kalemci, E., Tomsick, J. A., Buxton, M. M., et al. 2005, ApJ, 622, 508

Kalemci, E., Tomsick, J. A., Rothschild, R. E., et al. 2006, ApJ, 639, 340

Kylafis, N. D., Papadakis, I. E., Reig, P., Giannios, D., \& Pooley, G. G. 2008, A\&A, 489, 481

Kylafis, N. D., Contopoulos, I., Kazanas, D., \& Christodoulou, D. 2012, A\&A, 538, A5

Lasota, J.-P. 2001, New Astron. Rev., 45, 449

Levine, A. M., Bradt, H, Morgan, E. H., \& Remillard, R. 2006, Adv. Space Rev., 38, 2970

Liu, B. F., Yuan, W., Meyer, F., Meyer-Hofmeister, E., \& Xie, G. Z. 1999, ApJ, 527, L17

Lovelace, R. V. E., Rothstein, D. M., \& Bisnovatyi-Kogan, G. S. 2009, ApJ, 701,885

Lynden-Bell, D. 1996, MNRAS, 279, 389

Machida, M., Nakamura, K., \& Matsumoto, R. 2006, PASJ, 58, 193

Markoff, S., Nowak, M. A., \& Wilms, J. 2005, ApJ, 635, 1203

Marsh, T. R., Robinsin, E. L., \& Wood, J. H. 1994, MNRAS, 266, 137

McClintock, J. E., Narayan, R., Garcia, M. R., et al. ApJ, 593, 435

McClintock, J. E., Remillard, R. A., Rupen, M. P., et al. 2009, ApJ, 698, 1398

Meyer, F., \& Meyer-Hofmeister, E. 1981, A\&A, 104, L10

Meyer, F., Liu, B. F., \& Meyer-Hofmeister, E. 2000, A\&A, 361, 175

Meyer-Hofmeister, E., \& Meyer, F. 2001, A\&A, 380, 739

Meyer-Hofmeister, E., Liu, B. F., \& Meyer, F. 2009, A\&A, 508, 329

Mignone, A., Rossi, P., Bodo, G., Ferrari, A., \& Massaglia, S. 2010, MNRAS, 402, 7

Miller, J. M., Homan, J., \& Miniuti, G. 2006a, ApJ, 652, L113

Miller, J. M., Homan, J., Steeghs, D., et al. 2006b, ApJ, 653, 525

Miller-Jones, J. C. A., Sivakoff, G. R., Altamirano, D., et al. 2012, MNRAS, 421, 468

Miyamoto, S., Iga, S., Kitamoto, S., \& Kamado, Y. 1993, ApJ, 403, L39

Miyamoto, S., Kitamoto, S., Hayashida, K., \& Egoshi, W. 1995, ApJ, 442, L13

Motta, S., Belloni, T., \& Homan, J. 2009, MNRAS, 400, 1603

Motta, S., Muñoz-Darias, T., \& Belloni, T. 2010, MNRAS, 408, 1796

Motta, S., Muñoz-Darias, T., Casella, P., Belloni, T., \& Homan, J. 2011, MNRAS, 418, 2292

Motta, S., Homan, J., Muñoz-Darias, C., P., et al. 2012, MNRAS, 427, 595

Munoz-Darias, T., Motta, S., \& Belloni, T. M. 2011a, MNRAS, 410, 679

Munoz-Darias, T., Motta, S., Stiele, H., \& Belloni, T. M. 2011b, MNRAS, 415, 292

Narayan, R., \& Yi, I. 1994, ApJ, 428, L13

Narayan, R., \& Yi, I. 1995, ApJ, 452, 710

Narayan, R., McClintock, J. E., \& Yi, I. 1996, ApJ, 457, 821

Narayan, R., Barret, D., \& McClintock, J. E. 1997, ApJ, 482, 448

Ohsuga, K., Mineshige, S., Mori, M., \& Kato, Y. 2009, PASJ, 61, L7

Orosz, J. A., Bailyn, C. D., Relillard, R. A., McClintock, J. E., \& Foltz, C. B. 1994, ApJ, 436, 848

Plant, D. S., Fender, R. P., Ponti, G., Munoz-Darias, T., \& Coriat M. 2013, MNRAS, 442, 1767

Pottschmidt, K., Wilms, J., Nowak, M. A., et al. 2003, A\&A, 407, 1039

Poutanen, J., Krolik, J. H., \& Ryde, F. 1997, MNRAS, 292, L21

Reig, P., Kylafis, N. D., \& Giannios, D. 2003, A\&A, 403, L15

Qian, L., Liu, B. F., \& Wu, X.-B. 2007, ApJ, 669, 1145

Reis, R. C., Fabian, A. C., \& Miller, J. M. 2010, MNRAS, 402, 836

Remillard, R. A., \& McClintock, J. E. 2006, ARA\&A, 44, 49

Reynolds, M. T., \& Miller, J. M. 2013, ApJ, 769, 16

Romanova, M. M., Ustyugova, G. V., Koldova, A. V., \& Lovelace, R. V. E. 2009, in Protostellar Jets in Context, eds. K. Tsinganos, O. Ray, \& M. Stute (Berlin: Springer), Ap\&SS, Proc. 153

Shakura, N. I., \& Sunyaev, R. A. 1973, A\&A, 24, 337

Smak, J. 1984, Acta Astron., 34, 161

Sobolewska, M. A., Papadakis, I. E., Done, C., \& Malzac, J. 2011, MNRAS, 417,280

Stiele, H., Motta, S., Munoz-Darias, T., \& Belloni, T. M. 2011, MNRAS, 418, 1746

Stiele, H., Muñoz-Darias, T., Motta, S., \& Belloni, T. 2012, MNRAS, 422, 679

Tanaka, Y., \& Lewin, W. H. G. 1995, in X-ray Binaries, eds. W. H. G. Lewin, J. van Paradijs, \& E. P. J. van den Heuvel (Cambridge: Cambridge University Press), 126

Tchekhovskoy, A., Narayan, R., \& McKinney, J. C. 2011, MNRAS, 327, L79

Uttley, P., Wilkinson, T., Cassatella, P., et al. 2011, MNRAS, 414, L60

Wilkinson, T., \& Uttley, P. 2009, 397, 666 